\title{
ANALYTIC SOLUTION OF STOKES SECOND PROBLEM FOR SECOND-GRADE FLUID
}

\author{
S. ASGHAR, S. NADEEM, K. HANIF, AND T. HAYAT \\ Received 4 November 2004; Revised 28 July 2005; Accepted 26 October 2005
}

Using Laplace transformation and perturbation techniques, analytical solution is obtained for unsteady Stokes' second problem. Expressions for steady and transient solutions are explicitly determined. These solutions depend strongly upon the material parameter of second-grade fluid. It is shown that phase velocity decreases by increasing material parameter of second-grade fluid.

Copyright (c) 2006 S. Asghar et al. This is an open access article distributed under the Creative Commons Attribution License, which permits unrestricted use, distribution, and reproduction in any medium, provided the original work is properly cited.

\section{Basic equations}

We consider a semi-infinite expanse of homogeneous incompressible second-grade fluid which occupies the space above an infinitely extended plate in the $x z$-plane. Initially, the fluid is at rest and at time $t>0$ the plate starts oscillations in its own plane with velocity $U_{0} e^{i \omega t}$, where $\omega$ is the frequency of oscillating plate and $U_{0}$ is the constant velocity.

Viscoelastic fluids can be modeled by Rivlin-Ericksen constitutive equation

$$
\mathbf{T}=-p \mathbf{I}+\mu \mathbf{A}_{1}+\alpha_{1} \mathbf{A}_{2}+\alpha_{2} \mathbf{A}_{1}^{2}
$$

where $p$ is the pressure, $\mathbf{I}$ is the unit tensor, $\mathbf{T}$ is the Cauchy stress tensor, and $\mu, \alpha_{1}, \alpha_{2}$ are measurable material constants. They denote, respectively, the viscosity, elasticity, and cross-viscosity. These material constants can be determined from viscometric flows for any real fluid. $\mathbf{A}_{1}$ and $\mathbf{A}_{2}$ are Rivlin-Ericksen tensors and they denote, respectively, the rate of strain and acceleration. $\mathbf{A}_{1}$ and $\mathbf{A}_{2}$ are defined by

$$
\begin{gathered}
\mathbf{A}_{1}=(\operatorname{grad} \mathbf{V})+(\operatorname{grad} \mathbf{V})^{T}, \\
\mathbf{A}_{2}=\frac{d \mathbf{A}_{1}}{d t}+(\operatorname{grad} \mathbf{V})^{T} \mathbf{A}_{1}+\mathbf{A}_{1}(\operatorname{grad} \mathbf{V})
\end{gathered}
$$

where $d / d t$ is the material time derivative. The viscoelastic fluids when modeled by RivlinEricksen constitutive equation are termed as second-grade fluids. Second-grade fluids 
are dilute polymeric solutions (e.g., poly-iso-butylene, methyl-methacrylate in n-butyl acetate, polyethylene oxide in water, etc.). The equation is frame invariant and applicable for low shear rates. A detailed account on the characteristics of second-grade fluids is well documented by Dunn and Rajagopal [2]. Rajagopal and Gupta [7] have studied the thermodynamics in the form of dissipative inequality (Clausius-Duhem) and commonly accepted the idea that the specific Helmholtz free energy should be minimum in equilibrium. From the thermodynamics consideration they assumed

$$
\mu \geq 0, \quad \alpha_{1}>0, \quad \alpha_{1}+\alpha_{2}=0 .
$$

We seek the velocity field of the form

$$
(u(y, t), 0,0) .
$$

For this type of flow, equation of continuity is identically satisfied and the balance of linear momentum reduces to the following differential equation [5]:

$$
\frac{\partial u}{\partial t}=v \frac{\partial^{2} u}{\partial y^{2}}+\alpha \frac{\partial^{3} u}{\partial y^{2} \partial t}
$$

In the above $\nu=\mu / \rho$ is the kinematic viscosity, $\alpha=\alpha_{1} / \rho$, and $\rho$ is the density of the fluid. The proposed boundary and initial conditions for the problem are

$$
\begin{gathered}
u(y, t)=U_{0} e^{i \omega t}, \quad \text { at } y=0, t>0, \\
u(y, t) \longrightarrow 0, \quad \text { as } y \longrightarrow \infty, \forall t, \\
u(y, 0)=0, \quad y>0,
\end{gathered}
$$

where $\omega$ is the imposed frequency of oscillation.

In general, in the case of fluids of differential type, the equations of motion are of order higher than that of the Navier-Stokes equations, and thus the adherence boundary condition is insufficient to determine the solution completely (see [6-8] for detailed discussion of the relevant issues).

\section{Solution of the problem}

We will use the Laplace transform to study solutions to (1.5); with this technique the initial condition is automatically satisfied and the application of the boundary conditions is easier. However, the inversion procedure for obtaining the solution is not always straightforward and often requires care and ingenuity. The incompatibility between the initial and boundary conditions causes difficulties in establishing a smooth solution to the problem in the case of a fluid of grade two (see Bandelli et al. [1] for a detailed discussion of the relevant issue).

The Laplace transform pair is defined by

$$
\begin{gathered}
F(y, s)=\int_{0}^{\infty} u(y, t) e^{-s t} d t \\
u(y, t)=\frac{1}{2 \pi i} \int_{\lambda-i \infty}^{\lambda+i \infty} F(y, s) e^{s t} d s, \quad \lambda>0 .
\end{gathered}
$$


S. Asghar et al. 3

The problem in the transformed plane is given by

$$
\begin{gathered}
\alpha s \frac{d^{2} F}{d y^{2}}+v \frac{d^{2} F}{d y^{2}}-s F=0, \\
F(y, s)=\frac{U_{0}}{s-i \omega}, \quad \text { at } y=0, \\
F(y, s)=0, \quad \text { as } y \longrightarrow \infty .
\end{gathered}
$$

To solve (2.3), we use the regular perturbation and expand the function in terms of second-grade parameter $\alpha(\alpha \ll 1)$. That is,

$$
F=F_{0}+\alpha F_{1}+o\left(\alpha^{2}\right) .
$$

Substituting (2.5) into (2.3) and (2.4) and collecting the coefficients of equal powers of $\alpha$, we obtain

$$
\begin{gathered}
\nu \frac{d^{2} F_{0}}{d y^{2}}-s F_{0}=0, \\
\nu \frac{d^{2} F_{1}}{d y^{2}}-s F 1=-s \frac{d^{2} F_{0}}{d y^{2}}, \\
F_{0}=\frac{U_{0}}{s-i \omega}, \quad F_{1}=0, \quad \text { at } y=0, \\
F_{0}=F_{1}=0, \quad \text { as } y \longrightarrow \infty .
\end{gathered}
$$

The solution of the above differential systems can be easily written as

$$
F=\frac{U_{0}}{s-i \omega} e^{-\sqrt[y]{s / v}}\left[1+\frac{\alpha y}{2}\left(\frac{s}{v}\right)^{3 / 2}\right]
$$

Using (2.7) in (2.2) and then taking the inverse Laplace transform, we arrive at

$$
u(y, t)=\frac{U_{0}}{2}\left[\begin{array}{c}
\left(1+\frac{y \alpha}{2}\left(i \frac{\omega}{v}\right)^{3 / 2}\right) e^{-\sqrt[y]{i(w / v)}+i \omega t} \operatorname{erf} c\left(\frac{y}{2 \sqrt{v t}}-\sqrt{i \omega t}\right) \\
+\left(1-\frac{y \alpha}{2}\left(i \frac{\omega}{v}\right)^{3 / 2}\right) e^{y / \sqrt{i(w / v)}+i \omega t} \operatorname{erf} c\left(\frac{y}{2 \sqrt{v t}}+\sqrt{i \omega t}\right) \\
+\alpha y\left(\frac{y^{2}-2 v t+4 i \omega v t^{2}}{4 \sqrt{\pi(v t)^{5}}}\right) e^{-y^{2} / 4 v t}
\end{array}\right]
$$

where erf $c(x+i y)$ can be calculated in terms of tabulated functions [3]. The tables given in [3] do not give erf $c(x+i y)$ directly; therefore we define an auxiliary function $H(x+i y)$ as

$$
\operatorname{erf} c(x+i y)=e^{-(x+i y)^{2}} H(-y+i x)
$$


4 Analytic solution of Stokes second problem for second-grade fluid

where

$$
\begin{gathered}
H(-y+i x)=\overline{H(x+i y)}, \\
H(x-i y)=2 e^{-(x+i y)^{2}}-\overline{H(x+i y)},
\end{gathered}
$$

and $\overline{H(x+i y)}$ is the complex conjugate of $H(x+i y)$.

Introducing the nondimensional variables

$$
\eta=\frac{y U_{0}}{\nu}, \quad \tau=\frac{U_{0}^{2} t}{\nu}, \quad \beta=\frac{\alpha U_{0}^{2}}{v^{2}}, \quad \delta=\frac{\omega \nu}{U_{0}^{2}}, \quad u^{*}=\frac{u}{U_{0}}
$$

in (2.8), we obtain the velocity field as

$$
\frac{u(\eta, \tau)}{U_{0}}=\frac{1}{2}\left[\begin{array}{c}
\left(1+\eta \beta \delta^{3 / 2}(i)^{3 / 2}\right) e^{-((1+i) / \sqrt{2}) \eta \sqrt{\delta}+i \delta \tau} \operatorname{erf} c\left(\frac{\eta}{2 \sqrt{\tau}}-\sqrt{i \delta \tau}\right) \\
+\left(1-\eta \beta \delta^{3 / 2}(i)^{3 / 2}\right) e^{((1+i) / \sqrt{2}) \eta \sqrt{\delta}+i \delta \tau} \operatorname{erf} c\left(\frac{\eta}{2 \sqrt{\tau}}+\sqrt{i \delta \tau}\right) \\
+\beta \eta\left(\frac{\eta^{2}-2 \tau+4 i \delta \tau^{2}}{4 \sqrt{\pi \tau^{5}}}\right) e^{-\eta^{2} / 4 \tau}
\end{array}\right] .
$$

The solution given in (2.12) is general. For large times we must recover the steady-state solution. Indeed, when $\tau$ goes to infinity, we have

$$
\begin{aligned}
& \operatorname{erf} c\left(\frac{\eta}{2 \sqrt{\tau}}-\sqrt{i \tau}\right) \longrightarrow 2, \\
& \operatorname{erf} c\left(\frac{\eta}{2 \sqrt{\tau}}+\sqrt{i \tau}\right) \longrightarrow 0
\end{aligned}
$$

In view of the above results, (2.12) takes the following form:

$$
\frac{u_{s}}{U_{0}}=\left(1+\eta \beta \delta^{3 / 2}(i)^{3 / 2}\right) e^{-\eta \sqrt{\delta} / \sqrt{2}+i(\delta \tau-\eta \sqrt{\delta} / \sqrt{2})},
$$

where $u_{s}$ is the steady-state solution. For $\beta=0$, we recover the large time solution of Erdogan [4]. This confirms that for large times the starting solution tends to steadystate solution. It is clearly seen that since the steady-state solution is valid for large values of time only, it becomes independent of the initial condition. For some times after the initiation of the motion, the velocity field contains a transient which gradually disappears in time. The transient solution is obtained by subtracting (2.14) from (2.12); that is,

$$
\frac{u_{t}}{U_{0}}=\frac{1}{2}\left[\begin{array}{c}
\left(1+\eta \delta^{3 / 2} \beta(i)^{3 / 2}\right) e^{-((1+i) / \sqrt{2}) \eta+i \delta \tau}\left[\operatorname{erf} c\left(\frac{\eta}{2 \sqrt{\tau}}-\sqrt{i \delta \tau}\right)-2\right] \\
+\left(1-\eta \delta^{3 / 2} \beta(i)^{3 / 2}\right) e^{((1+i) / \sqrt{2}) \eta+i \delta \tau} \operatorname{erf} c\left(\frac{\eta}{2 \sqrt{\tau}}+\sqrt{i \delta \tau}\right) \\
+\beta \eta\left(\frac{\eta^{2}-2 \tau+4 i \delta \tau^{2}}{4 \sqrt{\pi \tau^{5}}}\right) e^{-\eta^{2} / 4 \tau}
\end{array}\right]
$$

where $u_{t}$ shows the transient solution. It can be seen clearly from (2.15) that for large times the transient solution disappears. From the steady-state solution equation (2.14) 
we can find the various physical quantities for the wave propagation in the second-grade fluid. The amplitude of the waves is given by

$$
R=e^{-\eta \sqrt{\delta} / \sqrt{2}} \sqrt{\left(1-\frac{\eta \beta \delta^{3 / 2}}{\sqrt{2}}\right)^{2}+\left(\frac{\eta \beta \delta^{3 / 2}}{\sqrt{2}}\right)^{2}}
$$

and the phase angle is

$$
\theta=\delta \tau-\frac{\eta \sqrt{\delta}}{\sqrt{2}}-\tan ^{-1} \phi, \quad \phi=\left(\frac{\eta \beta \delta^{3 / 2}}{\sqrt{2}+\eta \beta \delta^{3 / 2}}\right) .
$$

The phase velocity is

$$
v=\frac{\sqrt{2 \delta}\left(1+\eta^{2} \beta^{2} \delta^{3}+\sqrt{2} \eta \beta \delta^{3 / 2}\right)}{\left(1+\eta^{2} \beta^{2} \delta^{3}+\sqrt{2} \eta \beta \delta^{3 / 2}\right)+\beta \delta} .
$$

We can estimate the numerical value of the second-grade parameter $\beta$ through amplitude variation of the velocity. Let us define

$$
A(\eta)=\max u(\eta, \tau)-\min u(\eta, \tau) .
$$

Using (2.14), we can write $A(\eta)$ as

$$
A(\eta)=2 e^{-\eta \sqrt{\delta} / \sqrt{2}} \sqrt{\left(1-\frac{\eta \beta \delta^{3 / 2}}{\sqrt{2}}\right)^{2}+\left(\frac{\eta \beta \delta^{3 / 2}}{\sqrt{2}}\right)^{2}} .
$$

From (2.20) we obtain

$$
\frac{\mathbf{A}(\eta)}{\mathbf{A}(0)}=e^{-\eta \sqrt{\delta} / \sqrt{2}} \sqrt{\left(1-\frac{\eta \beta \delta^{3 / 2}}{\sqrt{2}}\right)^{2}+\left(\frac{\eta \beta \delta^{3 / 2}}{\sqrt{2}}\right)^{2}}=\frac{1}{2} \mathbf{A}(\eta) .
$$

Now, let $\eta_{1}$ be the depth for which $(1 / 2) A(\eta)$ falls to the value $1 / 2$. This gives

$$
e^{-\eta \sqrt{\delta} / \sqrt{2}} \sqrt{\left(1-\frac{\eta \beta \delta^{3 / 2}}{\sqrt{2}}\right)^{2}+\left(\frac{\eta \beta \delta^{3 / 2}}{\sqrt{2}}\right)^{2}}=\frac{1}{2} .
$$

From (2.22), we get

$$
\beta=\frac{\sqrt{\left(\sqrt{2} \eta_{1} \delta^{3 / 2}\right)^{2}-4 \eta_{1}^{2} \delta^{3 / 2}\left(1-(1 / 4) e^{2 \eta_{1} \sqrt{\delta / 2}}\right)}+\sqrt{2} \eta_{1} \delta^{3 / 2}}{2 \eta_{1}^{2} \delta^{3}} .
$$

\section{Discussion}

The effects of the second-grade parameter $(\beta)$ are clearly manifested through the following observations.

To discuss the nature of the velocity field, the real part of (2.12) and (2.14) are plotted against the distance $\eta$ from the plate. In Figure 3.1, the steady and total velocities are 
6 Analytic solution of Stokes second problem for second-grade fluid

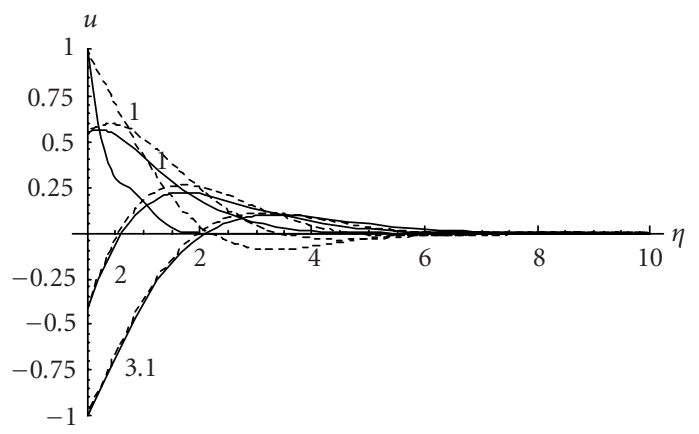

Figure 3.1. The variation in $u$ with $\eta$ for different values of $\tau$ at $\beta=0.1$ and $\delta=1$.

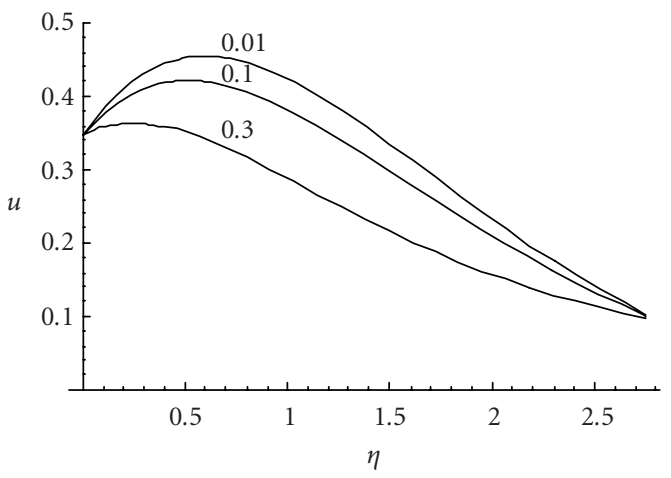

Figure 3.2. The variation in $u$ with $\eta$ for various values of $\beta$ for $\delta=1$ and $\tau=7.5$.

shown by dotted and continuous lines, respectively. It is observed that the steady-state is achieved at about $\tau=3.1$ for the second-grade parameter $\beta=0.1$. when $\beta$ is not equal to zero, the development of the fluid is retarded. This means $\beta$ will prolong the effects of transient state. The transient effect of second-grade fluid is greater than that of Newtonian fluid [4]. This observation clearly indicates the influence of second-grade fluid on the time taken to reach the steady-state. Figure 3.2 represents the effects of $\beta$ on velocity distribution and shows that the velocity decreases with the increase in $\beta$ and hence the boundary layer increases with the increase of $\beta$. The variation of the amplitude $\mathrm{R}$ with $\eta$ shows that increasing $\beta$ decreases the amplitude (see Figure 3.3). The effects of small $\delta$ on amplitude are shown in Figure 3.4. In Figure 3.5 phase angle $(\theta)$ is presented for different values of $\beta$. The figure shows that $\theta$ increases with the increase of $\beta$. The dependence of phase velocity (equation (2.18)) on the parameter $\beta$ is shown in Table 3.1 from which it is clearly seen that the phase velocity decreases with the increase of $\beta$. 


\section{S. Asghar et al. 7}

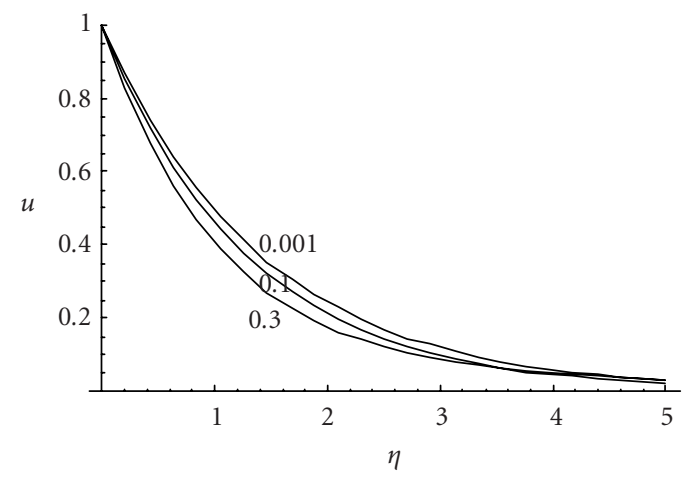

Figure 3.3. The variation in amplitude $R$ with $\eta$ for various values of $\beta$ for $\delta=1$.

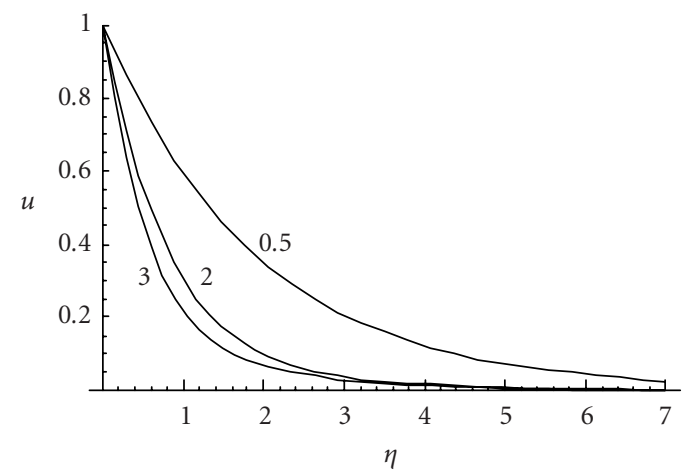

Figure 3.4. The variation in amplitude $R$ with $\eta$ for various values of $\delta$ for $\beta=0.1$.

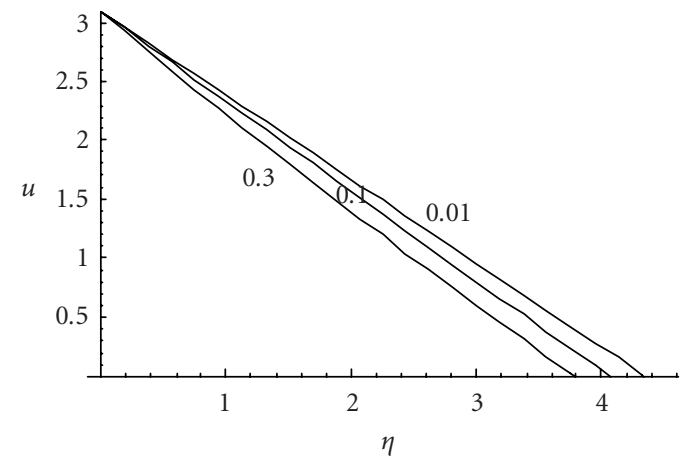

Figure 3.5. The variation in phase angle $\theta$ with $\eta$ for various values of $\beta$ for $\delta=1$ and $\tau=3.1$. 
8 Analytic solution of Stokes second problem for second-grade fluid

Table 3.1

\begin{tabular}{l|cccccc}
\hline$\eta \backslash \beta$ & 0 & 0.2 & 0.4 & 0.6 & 0.8 & 1 \\
\hline 0 & 1.414213 & 1.178511 & 1.010152 & 0.883883 & 0.785674 & 0.707106 \\
0.3 & 1.414213 & 1.194692 & 1.057112 & 0.964533 & 0.899254 & 0.851737 \\
0.6 & 1.414213 & 1.209863 & 1.099421 & 1.035189 & 0.996613 & 0.973446 \\
0.9 & 1.414213 & 1.223997 & 1.136742 & 1.094650 & 1.075138 & 1.067829 \\
\hline
\end{tabular}

\section{References}

[1] R. Bandelli, K. R. Rajagopal, and G. P. Galdi, On some unsteady motions of fluids of second grade, Archives of Mechanics 47 (1995), no. 4, 661-676.

[2] J. E. Dunn and K. R. Rajagopal, Fluids of differential type: critical review and thermodynamic analysis, International Journal of Engineering Science 33 (1995), no. 5, 689-729.

[3] A. Erdélyi, W. Magnus, F. Oberhettinger, and F. G. Tricomi, Higher Transcendental Functions, Bateman, Manuscript project, volume I, McGraw-Hill, New York, 1953.

[4] M. E. Erdogan, A note on an unsteady flow of a viscous fluid due to an oscillating plane wall, International Journal of Non-Linear Mechanics 35 (2000), no. 1, 1-6.

[5] C. Fetecau and J. Zierep, On a class of exact solutions of the equations of motion of a second grade fluid, Acta Mechanica 150 (2001), no. 1-2, 135-138.

[6] K. R. Rajagopal, On boundary conditions for fluids of the differential type, Navier-Stokes Equations and Related Nonlinear Problems (Funchal, 1994) (A. Sequira, ed.), Plenum, New York, 1995, pp. 273-278.

[7] K. R. Rajagopal and A. S. Gupta, An exact solution for the flow of a non-Newtonian fluid past an infinite porous plate, Meccanica 19 (1984), no. 2, 158-160.

[8] K. R. Rajagopal, A. Z. Szeri, and W. Troy, An existence theorem for the flow of a non-Newtonian fluid past an infinite porous plate, International Journal of Non-Linear Mechanics 21 (1986), no. 4, 279-289.

S. Asghar: Department of Mathematical Sciences, COMSATS Institute of Information Technology, Islamabad 44000, Pakistan

E-mail address: s_asgharpk@yahoo.com

S. Nadeem: Department of Mathematics, Quaid-i-Azam University, Islamabad 45320, Pakistan E-mail address: snqau@yahoo.com

K. Hanif: Department of Mathematics, Quaid-i-Azam University, Islamabad 45320, Pakistan E-mail address: khanif@yahoo.com

T. Hayat: Department of Mathematics, Quaid-i-Azam University, Islamabad 45320, Pakistan E-mail address: t_pensy@hotmail.com 


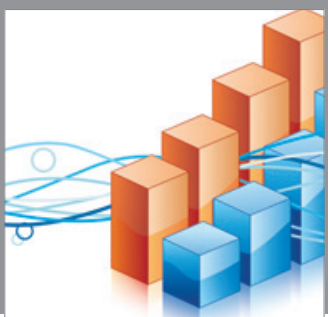

Advances in

Operations Research

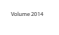

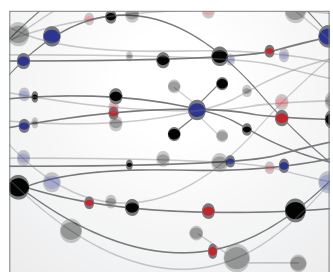

\section{The Scientific} World Journal
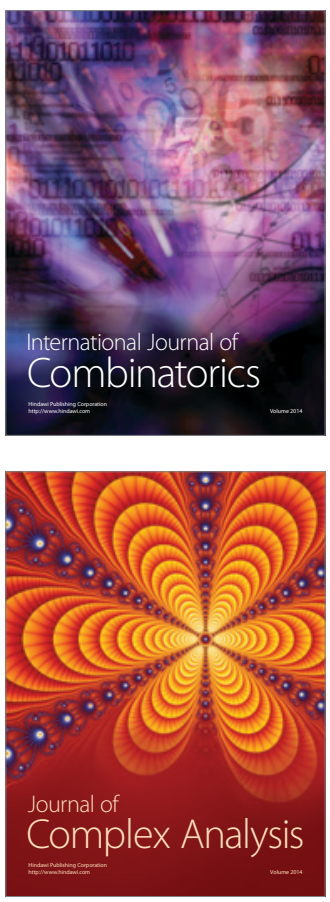

International Journal of

Mathematics and

Mathematical

Sciences
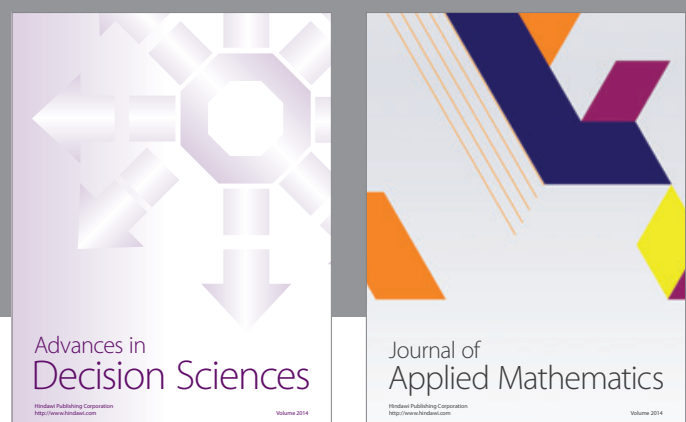

Journal of

Applied Mathematics
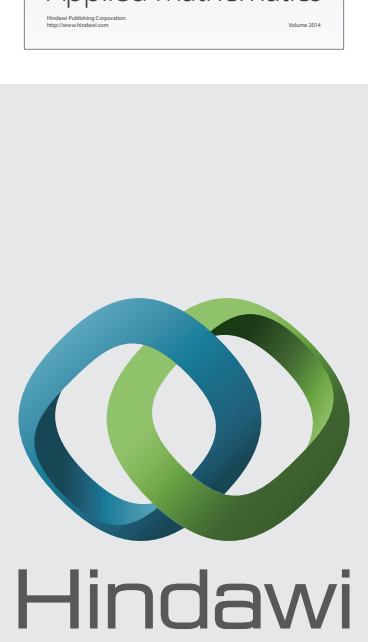

Submit your manuscripts at http://www.hindawi.com
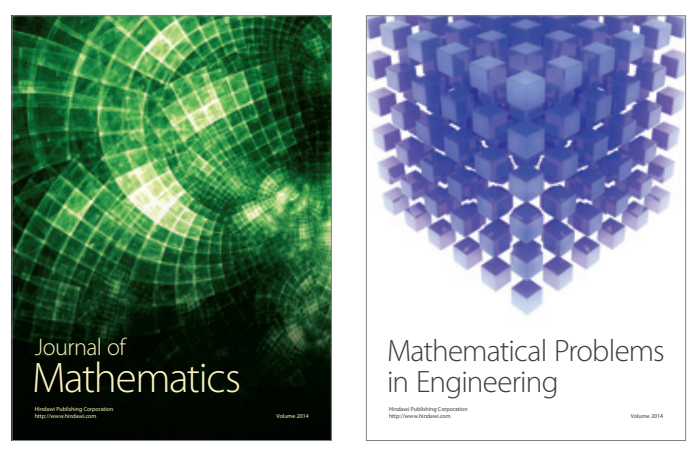

Mathematical Problems in Engineering
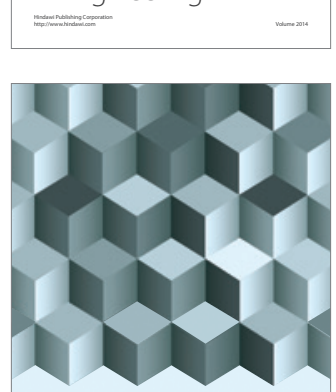

Journal of

Function Spaces
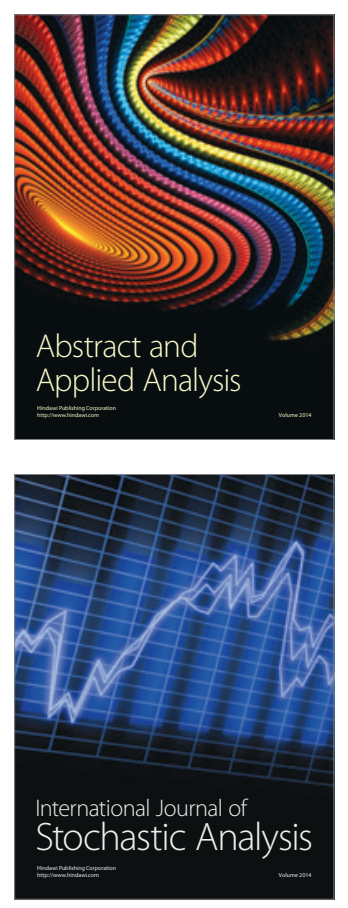

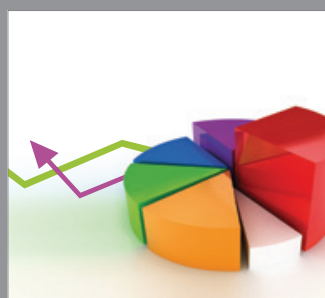

ournal of

Probability and Statistics

Promensencen
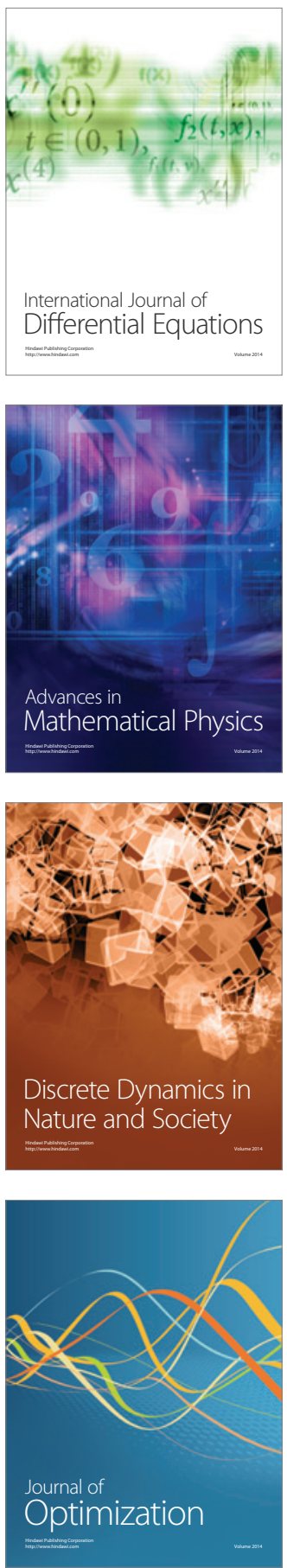